\title{
Automata for monadic second-order model-checking
}

\author{
Bruno Courcelle \\ Université Bordeaux-1, LaBRI, CNRS \\ 351, Cours de la Libération \\ 33405, Talence, France \\ courcell@labri.fr
}

We describe the construction of finite automata on terms establishing that the model-checking problem for every monadic second-order graph property is fixed-parameter linear for tree-width and clique-width (Chapter 6 of [6]).

In this approach, input graphs of small tree-width and clique-width are denoted by terms over finite signatures. These terms reflect the corresponding hierarchical decompositions and monadic second-order sentences are translated into automata intended to run on them. For the case of clique-width, this translation is a straightforward extension of that for finite words. It is a bit more complicated in the case of tree-width $([3],[4])$.

In both cases, the practical use of these constructions faces the problem that the automata are huge. (The number of states is typically an $h$-iterated exponential where $h$ is the quantifier alternation depth.) We present some tools (based on common work in progress with I. Durand, see [5]) that help to overcome this difficulty, at least in some cases. First we use automata whose states are described in an appropriate syntax (and not listed) and whose transitions are computed only when needed (and not compiled in unmanageable tables). In particular, automata are not systematically determinized. They can take as input terms denoting graphs having a tree-width or clique-width that is not a priori bounded. Our second tool consists in attaching to each position of the input term a contextual information (computed by one or two preliminary top-down and/or bottom-up passes), that helps to reduce the size of the automata.

The automata approach to monadic second-order model-checking is flexible in that it is not problem specific. Another similar flexible one, based on games, is developed by Kneiss et al. in [11].

The parsing problem consisting in checking that the tree-width or cliquewidth of a given graph is at most some given integer and in constructing a witnessing decomposition is also difficult ([1], [2], [9]), but we do not discuss it in this communication.

\section{References}

1. H. Bodlaender and A. Koster, Treewidth computations I. Upper bounds, Information and Computation 208 (2010) 259-275.

2. H. Bodlaender and A. Koster, Treewidth computations II. Lower bounds, Information and Computation, 2011, In Press. 
3. B. Courcelle, Special tree-width and the verification of monadic second-order graph properties, Foundations of Software Technology and Theoretical Computer Science, Chennai, India, 2010. Proceedings edited by K. Lodaya and M. Mahajan, LIPICs Vol. 8, pp. 13-29.

4. B. Courcelle, On the model-checking of monadic second-order formulas with edge set quantifications, May 2010, to appear in Discrete Applied Mathematics. Available from: http://hal.archives-ouvertes.fr/hal-00481735/fr/

5. B. Courcelle and I. Durand, Automata for the verification of monadic second-order graph properties, 2011, In preparation.

6. B. Courcelle and J. Engelfriet, Graph structure and monadic second-order logic, a language theoretic approach, 2011, To be published by Cambridge University Press. See: http://www.labri.fr/perso/courcell/Book/TheBook.pdf

7. B. Courcelle, J. Makowsky and U. Rotics, On the fixed parameter complexity of graph enumeration problems definable in monadic second-order logic, Discrete Applied Mathematics 108 (2001) 23-52.

8. R. Downey and M. Fellows, Parameterized complexity, Springer-Verlag, 1999

9. M. Fellows, F. Rosamond, U. Rotics and S. Szeider, Clique-width is NP-complete, SIAM J. Discrete Math. 23 (2009) 909-939.

10. J. Flum and M. Grohe, Parametrized complexity theory, Springer, 2006.

11. J. Kneiss, A. Langer, P. Rossmanith, Courcelle's Theorem - A Game-Theoretic Approach, ArXiv, CoRR abs/1104.3905, (2011) 